\title{
THE 1989 NOBEL PRIZE
}

\section{Ion Traps, an Isolated Electron and Atomic Clocks}

\author{
W. Schleich and $H$. Walther \\ Max-Planck-Institut für Quantenoptik, Garching, FRG
}

It has traditionally been the main endeavour of atomic spectroscopists to develop measurement techniques yielding ever higher resolutions, and to finally achieve the ultimate limit - the natural linewidth set by the spontaneous decay of energy levels. When long lifetimes are involved, as e.g. for hyperfine splittings of atomic ground states or of forbidden transitions, it is mostly the finite interaction time of the atom with the probing field which determines the linewidth. An extension of the interaction region is sometimes not a way out since additional problems may arise owing to field imperfections which usually become more significant as the interaction zone becomes larger.

There have been two developments which overcame this problem and extended resolution limits. The first is the invention of traps for charged particles together with new cooling techniques. These allow one to store a single charged particle for days and even months, and to cool it to temperatures in the milli-Kelvin regime. Together they eliminate transit time broadening as well as the quadratic Doppler effect which becomes important at resolutions in the range of $10^{12}$. The other approach uses two separated, phase coupled, measuring fields leading to an effective interaction time determined by the atom's time of flight between the two regions.

Professor Herbert Walther has been with the University of München since 1975 . He is also the Director of the Max-Planck-Institut für Quantenoptik (MPQ), Postfach 1513, D-8046 Garching, FRG and a scientific member of the Max-Planck-Gesellschaft. A graduate of the Universities of Heidelberg and Hannover, his main interests lie in quantum electronics. $\mathrm{He}$ has received several important awards, the latest being the American Optical Society's Charles Hard Townes Award for 1990.

Dr. Wolfgang Schleich is a graduate of the Ludwig Maximilians University, München where he received his final degree in 1989. A theoretician with MPQ, Garching since 1986 , his interests include general relatively and quantum phenomena. He has worked at the Centre for Theoretical Physics, Texas, and at the Centre for Advanced Studies, New Mexico.
The importance of these discoveries has been acknowledged by the decision of the Royal Swedish Academy of Sciences to award one half of the 1989 Nobel Prize in Physics to Professor Norman F. Ramsey, Harvard University, Cambridge, USA, for the invention of the separated oscillatory fields method and its use in the hydrogen maser and other atomic clocks; and one half jointly to Professor Hans G. Dehmelt, University of Washington, Seattle, USA, and Professor Wolfgang Paul, University of Bonn, FRG, for the development of the ion trap technique.

The present article illustrates the discoveries of the Nobel laureates by concentrating on a few highlights, but recognizing that they have contributed extensively to various branches of physics in ways that will not be discussed.

\section{The $g$-factor of the Electron}

In the late 1920's P.A.M. Dirac discovered a matter wave equation which, in contrast to the Schrödinger equation, satisfies the laws of special relativity. The equation predicts a $g$-factor for the electron of value 2. Physicists recognized in the 1940's that the vacuum of the microscopic world is by no means empty: the creation and annihilation of electron and positron pairs and fluctuations of electric and magnetic fields cause a slight departure of the $g$-factor of the electron from the value 2 . The first quantum electrodynamical correction was calculated by Julian Schwinger in 1949. Today, higher order corrections have been included giving $g / 2=1.001159652133$ (29). This deviation from Dirac's original theory triggered in the 1950's much work to test the QED prediction. A group at the University of Michigan led by H.C. Crane and A. Rich stored a beam of electrons in a magnetic bottle. An applied magnetic field causes the electron's spin axis to precess, forcing the electrons themselves to describe circular orbits. If $g$ were exactly 2 , the frequency of the spin precession and the orbit frequency would be the same. The experiment [1] therefore detects the difference between the two frequencies, i.e., g-2. Unfortunately, the accuracy of this method is limited by the presence of many interacting, high speed electrons.

To isolate an electron from all extraneous influences and hold it still for examination - that is the ultimate goal. Hans Dehmelt once said that he recognized this experimentalist's dream "the day I saw my teacher, Richard Becker, in his electricity and magnetism lecture draw a dot on the blackboard saying, "Here is an electron...'.". It was trap technology developed by groups led by Wolfgang Paul and Hans Dehmelt which made this dream come true.

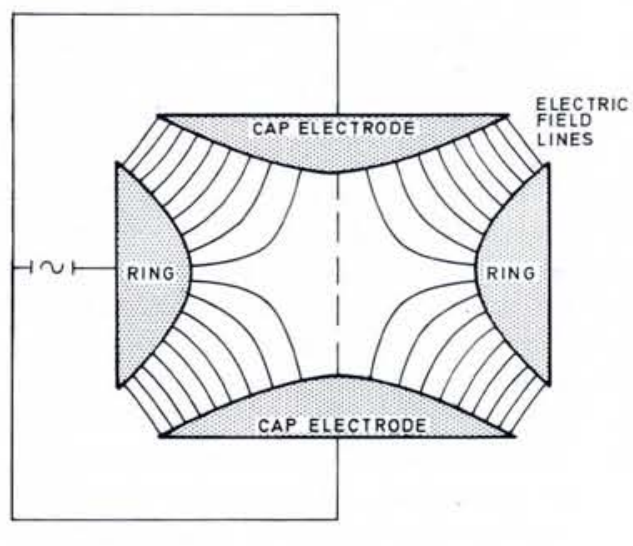

PAUL. TRAP

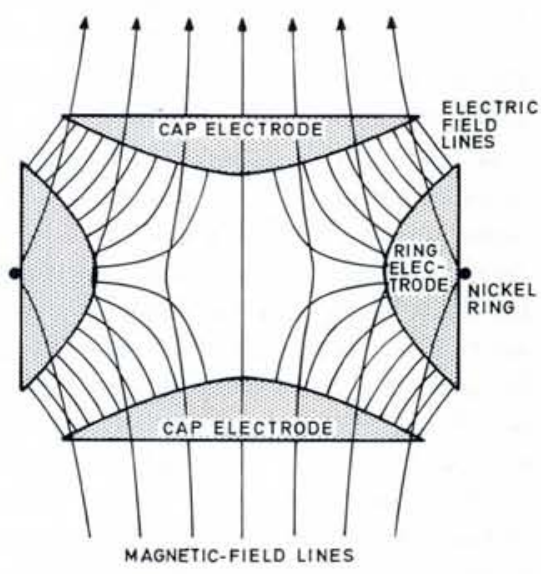

PENNING TRAP

Fig. 1 - Electrode arrangements of the Paul (left) and Penning (right) traps. 


\section{Trapping lons and Electrons}

In contrast to neutral atoms, ions can easily be influenced by electromagnetic fields because of their charge. In a first attempt towards trapping one can apply a constant voltage between a ring electrode and two end caps as shown in Fig. 1. This arrangement, however, does not achieve three-dimensional confinement. The potential that governs the ion's trajectory in the trap causes stable motion in the radial direction and unstable motion in the orthogonal direction, creating a saddle point at the trap centre. It is tempting to think that another, more sophisticated arrangement of electrodes would achieve three-dimensional confinement but the laws of electrostatics, and more precisely Poisson's equation, prevent this.

Fortunately there are at least two techniques that offer a way out of the problem. The first is based solely on the use of (time varying) electric fields. It is easily understood if we consider the saddle potential. As the particle starts to roll down the unstable hillside the sign of the voltage at the electrodes must be reversed so that the particle suddenly sees a rising rather than a decreasing potential, thus achieving dynamic binding. The motion of an ion in such a situation is described by the Mathieu differential equation, wellknown in classical mechanics, which, depending on the voltages applied to the trap, allows stable and unstable solutions. Under appropriate conditions one is able to obtain dynamic, threedimensional confinement in the trap. This type of "lonenkäfig" (ion cage) was conceived by the Nobel laureate Wolfgang Paul in 1955 and realized experimentally by his group [2]. The socalled Paul trap was based on Paul's earlier work with $\mathrm{H}$. Steinwedel and M. Raether demonstrating the use of a four-pole, time dependent electric field as a mass filter, a technique that is now the standard method for mass analysis and is also used extensively for trace analysis.

A different approach for obtaining three-dimensional confinement consists in superimposing on the electric field a constant, homogeneous, magnetic field aligned along the symmetry axis (Fig. 1, right-hand side). In the Penning trap the vertical magnetic field forces the charged particle to perform a circular motion in the plane orthogonal to the magnetic field, overruling the radial instability caused by the electrostatic field. While the ion oscillates back and forth along the symmetry axis of the trap it gyrates rapidly in small

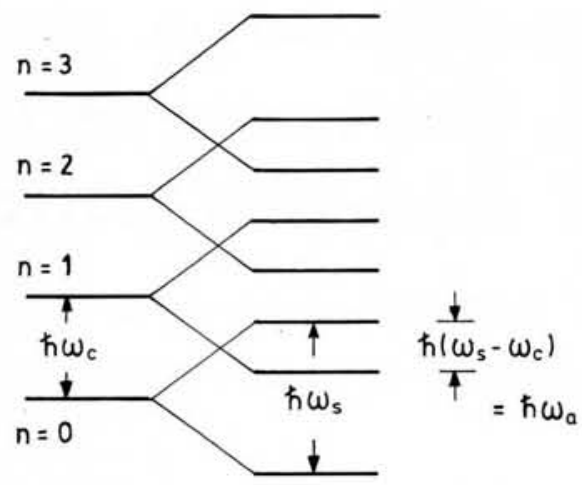

Fig. 2 - Energy levels of a trapped electron.

loops (the cyclotron motion); the centre of these loops progresses slowly around a larger circle (the magnetron motion).

Hans Dehmelt and his group were able to confine a single electron for months in such a trap. The set up in fact represented an artificial atom of macroscopic dimensions, namely an electron tied to an extraordinarily massive nucleus - the earth - which has led to it being called a geonium.

\section{The Geonium Experiment}

Quantum mechanics restricts the energy of an electron circulating in a magnetic field to discrete, equally spaced levels as shown on the left-hand side of Fig. 2. The frequency difference $\omega_{c}$ between consecutive energy levels of the electron's cyclotron motion, that is, between two adjacent cyclotron orbits is $\omega_{C}=2 \mu_{B} B / \hbar$ where $B$ denotes the magnetic field and $\mu_{B}$ is the Bohr magneton. The two possible spin orientations, spin parallel and antiparallel to the magnetic field, split each energy level into two energies with a frequency separation $\omega_{s}=g \mu_{B} B / \hbar$.

The energy difference $\hbar \omega_{a}$ (Fig. 2 ) is proportional to $(g-2)$ in that $\hbar \omega_{a}=$ $\hbar\left(\omega_{s}-\omega_{c}\right)=(g-2) \mu_{B} B$. The transition with frequency $\omega_{a}$ is a forbidden transition for the "geonium" but nevertheless it can be measured. When we divide $\omega_{a}$ by the cyclotron frequency, i.e., $\omega_{a} / \omega_{c}{ }_{c}$ $=(g-2) / 2$, the dependence on the magnetic field $B$ and the Bohr magneton can be eliminated. Hence, by measuring the frequencies $\omega_{a}$ and $\omega_{c}$ one can determine the quantity $(g-2) / 2$, giving the quantum electrodynamical correction to the $g$-factor directly. The transition is measured by observing the axial oscillation frequency of the electron. Owing to a small inhomogeneity of the magnetic field (produced by a nickel wire in the ring electrode, Fig. 1) and the continuous Stern-Gerlach effect, the axial fre- quency of the electron depends on its spin orientation. The spin-flip that takes place when the transition with $\omega_{a}$ is induced causes a detectable change of the axial oscillation frequency. The impressive result of these experiments and their successors [1] is a value of $g / 2=1.001159652188$ (4), equivalent to 4 parts in $10^{12}$. When we compare it to the quantum electrodynamical result we find agreement to one part in $10^{10}$.

If space permitted it would be tempting to stroll from the possibilities of performing spectroscopy on an isolated ion first demonstrated by Hans Dehmelt and $\mathrm{P}$. Toschek to the precision experiments with $\mathrm{mHz}$ resolution, or the observation of quantum jumps [3] and phase transitions of a few ions caught in a trap [4]. However, in the next section we focus on two clocks of unprecedented level of precision, the hydrogen maser and the cesium clock developed by N. Ramsey and collaborators [5].

\section{The Hydrogen Maser}

One of the essential ingredients of the hydrogen maser constructed for the first time by Norman Ramsey and D. Kleppner is a magnetic hexapole lens invented in 1951 by Wolfgang Paul and $\mathrm{H}$. Friedburg. Hydrogen atoms passing the hexapole field are selected according to their ground state hyperfine states: the $F=1$ atoms are focussed and the $F=0$ defocussed resulting in the population inversion of the two hyperfine states required for maser operation.

The hydrogen maser gives remarkably precise values of the hyperfine splitting of the hydrogen ground state (in $\mathrm{Hz}$ for ${ }^{1} \mathrm{H}$ : $1420405751.768 \pm 0.002$; for ${ }^{2} \mathrm{H}: 327384352.5222 \pm 0.0024$. It possesses a considerably higher frequency stability than the cesium clock for short and intermediate times (hours - days) but its absolute accuracy is inferior so it has been mainly employed as a secondary standard. For example, it has been used to measure the continental drift between Europe and the USA as being $17 \pm 3 \mathrm{~mm} /$ year. This value was obtained by receiving signals from an astronomical pulsar by means of radio telescopes placed at various locations on the two continents; the relative timing of the signals was determined using hydrogen masers [6].

Another application is the verification of the gravitational red shift. By comparing the frequencies of rocket-borne and earth-bound hydrogen masers, the prediction of general relativity has been verified to seven parts in $10^{5}$. 

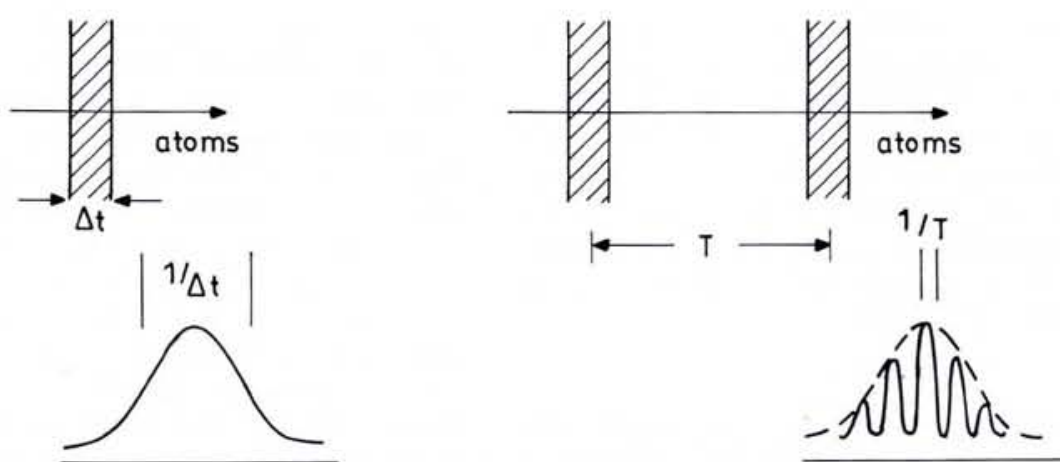

Fig. 3 - The Ramsey technique for two probing fields (right) as compared with the conventional one-field method (left). The left-hand hatched area symbolizes an atom-field interaction region with an interaction time $\Delta t$ while $T$ is the time-of-flight between two regions for the two-field arrangement. The two-field frequency spectrum is analogous to a two-slit interference pattern. Its envelope (dashed line) is equivalent to the one-field spectrum.

In Ramsey's two-field method, the atoms under investigation interact with two fields which are phase coupled (see Fig. 3). Owing to the coherent action of the two fields, the resonance curve is changed and shows an additional "interference" structure. The width of the central narrow peak is determined by the atom's time of flight between the two interaction regions.

The cesium clock, which has been used as a time standard since 1967, is based on this principle. Cesium-133 atoms in a beam are state selected according to their hyperfine states (as in the hydrogen maser). Transitions between the hyperfine levels are induced in the two-field interaction region and detected by means of a second hexapole magnet and a detector. According to the adopted definition of the time standard, the peak of the resonance curve (i.e. the hyperfine splitting), corresponds to exactly $9192631770 \mathrm{~Hz}$.

The two-field method is the basis of experiments aiming to determine the electric dipole moment of the neutron that are currently being undertaken by Norman Ramsey and collaborators at the ILL, Grenoble. The accuracy of the

\section{The 1989 Nobel Laureates}
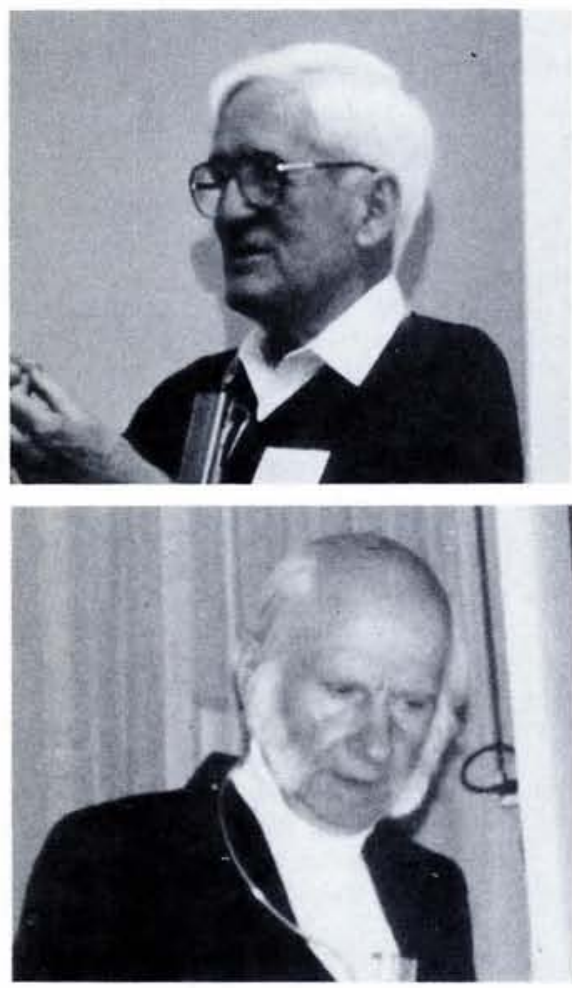

The 1989 Nobel laureates:Norman Ramsey (top), Hans Dehmelt (bottom) and Wolfgang Paul (top right).

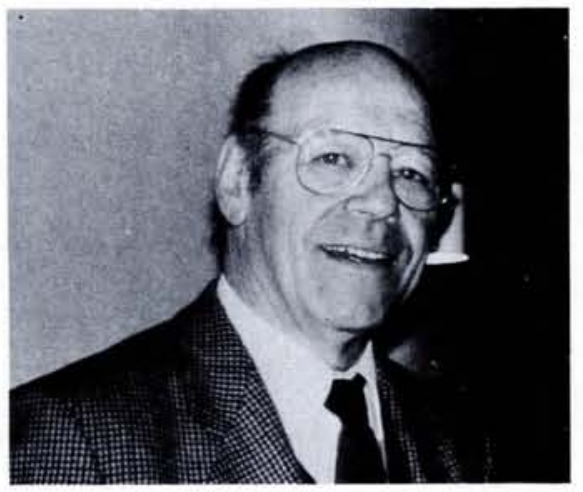

Norman Ramsey was appointed to the faculty of Harvard University in 1947 and retired recently as the Higgins Professor of Physics. He remains very active in lecturing and research, commuting regularly to the ILL, Grenoble where he continues his collaboration using the Institute's cold neutron facility. A graduate of Columbia University, he received his Ph.D. in 1940. After working at the MIT Radiation Laboratory and on the Manhattan Project, he returned to Columbia as an associate professor. He helped found Associate Universities Inc., the group that created the experiment at the present time gives an upper limit of $5 \times 10^{-25} \mathrm{e.cm}$ for the electric dipole moment.

The Nobel laureates of 1989 have clearly set the stage for a series of precision experiments in atomic spectroscopy. The field is still undergoing rapid development so further interesting results and applications relating to precision measurements must be expected in the near future.

\section{FURTHER READING}

[1] For a review of the history of the $g-2$ experiments, see: Dehmelt H.G. in: Advances in Laser Spectroscopy, Eds. F.T. Arecchi, F. Strumia and $\mathrm{H}$. Walther (Plenum Press, New York) 1983; Ekstrom P. and Wineland D., Scientific American 243 (1980) 104.

[2] For the original description of the Paul trap: Paul W., Osberghaus $\mathrm{O}$. and Fischer E., Ein lonenkäfig (Forschungsberichte des Wirtschafts- und Verkehrsministeriums, Nordrhein-Westfalen) 1958.

[3] On ion traps, laser cooling, quantum jumps, etc.: Itano W.M., Bergquist J.C. and Wineland D.J., Science 237 (1987) 612.

[4] On phase transitions and chaos: Quint W., Schleich W. and Walther H., Physics World 2 (1989) 30 and La Recherche 20 (1989) 1194.

[5] On Ramsey's method: Ramsey N.F., Physics Today (July 1980) 25.

[6] On atomic clocks: Hellwig H., Evenson K.M. and Wineland D.J., Physics Today (December 1978) 23.

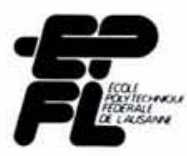

The group of electronic ceramics at the Swiss Federal Institute of Technology in Lausanne has the following openings:

Research associates (post-doctoral fellows):

1. Electrical properties of ferroelectrics and high- $K$ dielectrics

2. Processing of ferroelectric ceramics

3. Crystallographic study of electronic materials

Doctorate students:

1. Ferroelectric materials

2. Microwave ceramics

Submit applications to:

Prof. N. Setter

Département des matériaux EPFL

34, chemin de Bellerive

CH - 1007 Lausanne

Switzerland 